\title{
CHARACTERIZATIONS OF DISJOINTNESS PRESERVING OPERATORS ON VECTOR-VALUED FUNCTION SPACES
}

\author{
JYH-SHYANG JEANG AND YING-FEN LIN
}

(Communicated by Joseph A. Ball)

\begin{abstract}
We characterize compact and completely continuous disjointness preserving linear operators on vector-valued continuous functions as follows: a disjointness preserving operator $T: C_{0}(X, E) \rightarrow C_{0}(Y, F)$ is compact (resp. completely continuous) if and only if

$$
T f=\sum_{n} \delta_{x_{n}} \otimes h_{n}(f) \text { for all } f \in C_{0}(X, E),
$$

where $h_{n}: Y \rightarrow B(E, F)$ is continuous and vanishes at infinity in the uniform (resp. strong) operator topology, and $h_{n}(y)$ is compact (resp. $h_{n}$ is uniformly completely continuous).
\end{abstract}

\section{INTRODUCTION}

Let $X$ be a locally compact Hausdorff space, and let $E$ be a real or complex Banach space. Let $C_{0}(X, E)$ be the Banach space of all continuous $E$-valued functions on $X$, vanishing at infinity and equipped with the supremum norm. We write $C(X, E)$ instead of $C_{0}(X, E)$ in case $X$ is compact. For each $f$ in $C_{0}(X, E)$, the cozero of $f$, denoted by $\operatorname{coz}(f)$, is defined to be the open set $\operatorname{coz}(f)=\{x \in X: f(x) \neq$ $0\}$. A linear operator $T$ from $C_{0}(X, E)$ into $C_{0}(Y, F)$ is disjointness preserving if $T$ preserves disjointness of cozeros of functions, that is, $\operatorname{coz}(T f) \cap \operatorname{coz}(T g)=\emptyset$ whenever $\operatorname{coz}(f) \cap \operatorname{coz}(g)=\emptyset$. Equivalently, $\|T f(y)\|\|T g(y)\|=0$ for all $y \in Y$ whenever $\|f(x)\|\|g(x)\|=0$ for all $x \in X$.

Disjointness preserving operators between general vector lattices were considered by several authors (see, e.g., [2, 1, 4]). Lately such operators were studied between the spaces of real or complex-valued continuous functions under the name of separating operators (see, e.g., $[8,5]$ ), or between Fourier algebras (e.g. [6]). It was shown that a bounded disjointness preserving operator is a weighted composition operator. In the recent paper [9], a concrete representation is given for compact, weakly compact and completely continuous disjointness preserving operators from $C_{0}(X)$ into $C_{0}(Y)$.

Received by the editors August 4, 2006 and, in revised form, November 11, 2006.

2000 Mathematics Subject Classification. Primary 47B07, 47B38.

Key words and phrases. Compact operators, completely continuous operators, disjointness preserving operators.

The authors were partially supported by Taiwan NSC grants NSC94-2115-M-026-2116 and NSC94-2115-M-145-001.

The second author was supported by PIMS PDFs and was visiting the University of Alberta when this work was completed. 
Jamison and Rajagopalan in [7] studied disjointness preserving operators on vector-valued continuous functions. They gave a necessary and sufficient condition for such operators to be compact. In this paper, we shall give a representation of disjointness preserving operators $T: C_{0}(X, E) \rightarrow C_{0}(Y, F)$ that are compact or completely continuous. Indeed, such an operator $T$ can be written as a countable sum of atoms $\delta_{x_{n}} \otimes h_{n}$ with some corresponding properties.

\section{Characterizations of the operator $\delta_{x} \otimes h$}

Let $x$ be a fixed point in $X$ and $\delta_{x}$ be the point evaluation. Let $h$ be a map from $Y$ into $B(E, F)$, the Banach space of all bounded linear operators from $E$ into $F$. A linear map $\delta_{x} \otimes h$ sending $E$-valued functions on $X$ to $F$-valued functions on $Y$ is defined by

$$
\delta_{x} \otimes h(f)(y)=h(y)(f(x)) \text { for all } y \in Y .
$$

Let $(B(E, F), \mathrm{SOT})$ be the locally convex space with the strong operator topology. The map $h$ is said to be continuous in the strong operator topology if $h: Y \rightarrow$ $(B(E, F), \mathrm{SOT})$ is continuous. If $h: Y \rightarrow(B(E, F)$, SOT $)$ vanishes at infinity, we say that $h$ vanishes at infinity in the strong operator topology. Similarly, we say that $h: Y \rightarrow B(E, F)$ is continuous in the uniform operator topology when we consider $B(E, F)$ as a Banach space.

The following observation follows immediately from the Closed Graph Theorem.

Proposition 2.1. $\delta_{x} \otimes h$ maps $C_{0}(X, E)$ into $C_{0}(Y, F)$ if and only if $h$ is continuous and vanishes at infinity in the strong operator topology. Moreover, $\left\|\delta_{x} \otimes h\right\|=$ $\sup _{y \in Y}\|h(y)\|$, and the linear map $\delta_{x} \otimes h: C_{0}(X, E) \rightarrow C_{0}(Y, F)$ is automatically bounded.

In the following, we characterize the compactness and complete continuity of the bounded linear operator $\delta_{x} \otimes h$ from $C_{0}(X, E)$ into $C_{0}(Y, F)$. In case $X$ and $Y$ are compact, the following lemma was given in [3, Theorem 2.1] by a different approach.

Lemma 2.2. The bounded linear operator $\delta_{x} \otimes h$ from $C_{0}(X, E)$ into $C_{0}(Y, F)$ is compact if and only if $h: Y \rightarrow B(E, F)$ is continuous and vanishes at infinity in the uniform operator topology and $h(y)$ is a compact operator for each $y$ in $Y$.

Proof. For the necessity, it is clear that for every $y$ in $Y$, the bounded linear operator $h(y)$ is compact. Suppose that $h$ was not continuous at some point $y_{0} \in Y$ in the uniform operator topology. There exists an $\epsilon>0$, a net $\left\{y_{\lambda}\right\}_{\lambda}$ converging to $y_{0}$ in $Y$ and a net $\left\{e_{\lambda}\right\}_{\lambda}$ in $E$ with $\left\|e_{\lambda}\right\|=1$ for all $\lambda$ such that $\left\|h\left(y_{\lambda}\right)\left(e_{\lambda}\right)-h\left(y_{0}\right)\left(e_{\lambda}\right)\right\| \geq \epsilon$ for all $\lambda$. Let $\left\{f_{\lambda}\right\}_{\lambda}$ be in $C_{0}(X, E)$ such that $f_{\lambda}(x)=e_{\lambda}$ and $\left\|f_{\lambda}\right\|=1$. By the compactness of $\delta_{x} \otimes h$ and passing to a subnet, we can assume that $\delta_{x} \otimes h\left(f_{\lambda}\right)$ converges to some $g$ in $C_{0}(Y, F)$. Then $\left\|\delta_{x} \otimes h\left(f_{\lambda}\right)-g\right\|<\epsilon / 3$ for all $\lambda$ eventually, and we have that $\left\|h(y)\left(e_{\lambda}\right)-g(y)\right\|=\left\|\delta_{x} \otimes h\left(f_{\lambda}\right)(y)-g(y)\right\|<\epsilon / 3$ for all $y \in Y$ and all $\lambda$ eventually. Since $g$ is in $C_{0}(Y, F)$, we have

$$
\begin{aligned}
& \left\|h\left(y_{\lambda}\right)\left(e_{\lambda}\right)-h\left(y_{0}\right)\left(e_{\lambda}\right)\right\| \\
& \quad \leq\left\|h\left(y_{\lambda}\right)\left(e_{\lambda}\right)-g\left(y_{\lambda}\right)\right\|+\left\|g\left(y_{\lambda}\right)-g\left(y_{0}\right)\right\|+\left\|g\left(y_{0}\right)-h\left(y_{0}\right)\left(e_{\lambda}\right)\right\|<\epsilon
\end{aligned}
$$

for all $\lambda$ eventually, a contradiction. Therefore, $h$ is continuous on $Y$ in the uniform operator topology. By a similar argument as above, we have that $h$ vanishes at infinity in the uniform operator topology. 
For the sufficiency, let $\left\{f_{n}\right\}_{n}$ be in $C_{0}(X, E)$ with $\left\|f_{n}\right\|=1$ and let $\mathcal{U}$ be an ultrafilter in $\mathbb{N}$. For each $y$ in $Y$, by the compactness of $h(y)$, we have that $g(y)=\lim _{\mathcal{U}} h(y)\left(f_{n}(x)\right)$ exists. It is sufficient to show that $g$ is in $C_{0}(Y, F)$. For each $y_{0}$ in $Y$, since

$$
\begin{aligned}
\left\|g(y)-g\left(y_{0}\right)\right\| & =\lim _{\mathcal{U}}\left\|h(y)\left(f_{n}(x)\right)-h\left(y_{0}\right)\left(f_{n}(x)\right)\right\| \\
& \leq \lim _{\mathcal{U}}\left\|h(y)-h\left(y_{0}\right)\right\|\left\|f_{n}(x)\right\| \leq\left\|h(y)-h\left(y_{0}\right)\right\|
\end{aligned}
$$

and $h$ is continuous on $Y$ in the uniform operator topology, this implies that $g$ is continuous at $y_{0}$. It remains to show that $g$ vanishes at infinity. Since $\|h(y)\|$ vanishes at infinity, for every $\epsilon>0$, there is a compact subset $K_{\epsilon}$ of $Y$ such that $\|h(y)\|<\epsilon$ for all $y \notin K_{\epsilon}$. We have that $\|g(y)\|=\lim _{\mathcal{U}}\left\|h(y)\left(f_{n}(x)\right)\right\|<\epsilon$ for all $y \notin K_{\epsilon}$. Therefore, $g$ is in $C_{0}(Y, F)$.

Recall that a bounded linear operator $T: C_{0}(X, E) \rightarrow C_{0}(Y, F)$ is completely continuous if $\left\{T f_{n}\right\}_{n}$ is a null sequence for every weakly null sequence $\left\{f_{n}\right\}_{n}$ in $C_{0}(X, E)$. Let $h: Y \rightarrow B(E, F)$ be continuous and vanishing at infinity in the strong operator topology. We say that $h$ is uniformly completely continuous on $Y$ if, for every weakly null sequence $\left\{e_{n}\right\}_{n}$ in $E,\left\{h(\cdot)\left(e_{n}\right)\right\}_{n}$ is a uniformly null sequence.

Lemma 2.3. The bounded linear operator $\delta_{x} \otimes h: C_{0}(X, E) \rightarrow C_{0}(Y, F)$ is completely continuous if and only if $h$ is uniformly completely continuous on $Y$.

Proof. The necessity is trivial. For the sufficiency, if $\left\{f_{n}\right\}_{n}$ is a weakly null sequence in $C_{0}(X, E)$, then the sequence $\left\{e_{n}\right\}_{n}=\left\{f_{n}(x)\right\}_{n}$ is weakly null in $E$. Since $h$ is uniformly completely continuous, for each $\epsilon>0$, there is a positive integer $N_{\epsilon}$ such that

$$
\begin{aligned}
\left\|\delta_{x} \otimes h\left(f_{n}\right)\right\| & =\sup _{y \in Y}\left\|\delta_{x} \otimes h\left(f_{n}\right)(y)\right\| \\
& =\sup _{y \in Y}\left\|h(y)\left(e_{n}\right)\right\|<\epsilon \quad \text { for all } n \geq N_{\epsilon} .
\end{aligned}
$$

Hence $\delta_{x} \otimes h$ is completely continuous.

Corollary 2.4. Suppose that $h: Y \rightarrow B(E, F)$ is continuous and vanishes at infinity in the uniform operator topology, and $h(y)$ is completely continuous for every $y$ in $Y$. Then $h$ is uniformly completely continuous on $Y$. Consequently, the bounded linear operator $\delta_{x} \otimes h$ is completely continuous.

Proof. Let $\left\{e_{n}\right\}_{n}$ be a weakly null sequence in $E$. Without loss of generality, we can assume that $\left\|e_{n}\right\| \leq 1$ for all $n$. For every $\epsilon>0$, let $K_{\epsilon}$ be a compact subset of $Y$ such that $\|h(y)\|<\epsilon$ for all $y \notin K_{\epsilon}$. For each $y \in Y$, the set $U_{y}=\left\{y^{\prime} \in Y: \| h\left(y^{\prime}\right)-\right.$ $h(y) \|<\epsilon / 2\}$ is open in $Y$ by the continuity of $h$. There are finitely many points $y_{1}, y_{2}, \ldots, y_{k}$ in $K_{\epsilon}$ such that $K_{\epsilon} \subseteq \bigcup_{i=1}^{k} U_{y_{i}}$. For each $i=1,2, \ldots, k$, since $h\left(y_{i}\right)$ is completely continuous, there is a positive integer $N_{i}$ such that $\left\|h\left(y_{i}\right)\left(e_{n}\right)\right\|<\epsilon / 2$ for all $n \geq N_{i}$. Let $N=\max \left\{N_{1}, \ldots, N_{k}\right\}$. Then for each $y \in K_{\epsilon}$, we have $y \in U_{y_{i}}$ for some $y_{i}$ in $Y$, and $\left\|h(y)\left(e_{n}\right)\right\| \leq\left\|h(y)\left(e_{n}\right)-h\left(y_{i}\right)\left(e_{n}\right)\right\|+\left\|h\left(y_{i}\right)\left(e_{n}\right)\right\|<\epsilon$ for all $n \geq N$. On the other hand, for all $y \notin K_{\epsilon}$, we have $\left\|h(y)\left(e_{n}\right)\right\| \leq\|h(y)\|\left\|e_{n}\right\|<\epsilon$ for all $n$. Hence $h$ is uniformly completely continuous on $Y$. By Lemma 2.3, $\delta_{x} \otimes h$ is completely continuous. 
To close this section, we give a parallel result for the operator $\delta_{x} \otimes h$ being weakly compact. In the case that $X$ and $Y$ are compact, it was given in [3, Theorem 3.1] by a different approach.

Lemma 2.5. Let $h: Y \rightarrow B(E, F)$ be continuous and vanishing at infinity in the uniform operator topology. If $h(y)$ is weakly compact for every $y$ in $Y$, then $\delta_{x} \otimes h$ is a weakly compact operator from $C_{0}(X, E)$ into $C_{0}(Y, F)$.

Proof. Let $\left\{f_{n}\right\}_{n}$ be a sequence in $C_{0}(X, E)$ with $\left\|f_{n}\right\|=1$. Then the sequence $\left\{e_{n}\right\}_{n}=\left\{f_{n}(x)\right\}_{n}$ is bounded in $E$ with $\left\|e_{n}\right\| \leq 1$. Let $\mathcal{U}$ be an ultrafilter in $\mathbb{N}$. Since $h(y)$ is weakly compact for each $y \in Y$, we have that $g(y)=$ wk- $\lim _{\mathcal{U}} h(y)\left(e_{n}\right)$ exists in $F$. It is sufficient to show that $g$ is in $C_{0}(Y, F)$. For every $v^{*} \in F^{*}$, the dual space of $F$,

$$
\begin{aligned}
\left|v^{*}(g(y))-v^{*}\left(g\left(y_{0}\right)\right)\right| & =\lim _{\mathcal{U}}\left|v^{*}\left(h(y)\left(e_{n}\right)\right)-v^{*}\left(h\left(y_{0}\right)\left(e_{n}\right)\right)\right| \\
& \leq\left\|v^{*}\right\|\left\|h(y)-h\left(y_{0}\right)\right\|
\end{aligned}
$$

and

$$
\left|v^{*}(g(y))\right|=\lim _{\mathcal{U}}\left|v^{*}\left(h(y)\left(e_{n}\right)\right)\right| \leq\left\|v^{*}\right\|\|h(y)\| .
$$

We have $\left\|g(y)-g\left(y_{0}\right)\right\| \leq\left\|h(y)-h\left(y_{0}\right)\right\|$ and $\|g(y)\| \leq\|h(y)\|$. Hence, $g$ is in $C_{0}(Y, F)$ followed from the assumption of $h$.

\section{Compact and completely continuous DISJOINTNESS PRESERVING OPERATORS}

Let $T$ be a disjointness preserving bounded linear operator from $C_{0}(X, E)$ into $C_{0}(Y, F)$. Set $Y_{\infty}=\left\{y \in Y \cup\{\infty\}: \delta_{y} \circ T=0\right\}$ and $Y^{\prime}=\left\{y \in Y \cup\{\infty\}: \delta_{y} \circ T \neq 0\right\}$. From [3], such an operator $T$ can be represented as, for all $f \in C_{0}(X, E)$,

$$
\left.T f\right|_{Y_{\infty}} \equiv 0 \text { and } T f(y)=h(y)(f(\varphi(y))) \text { for all } y \text { in } Y^{\prime} \text {, }
$$

where $\varphi: Y^{\prime} \rightarrow X$ is continuous and $h: Y^{\prime} \rightarrow B(E, F)$ is continuous and vanishes at infinity in the strong operator topology. Hence, for each $x$ in $X$, the linear operator $\delta_{x} \otimes h: C_{0}(X, E) \rightarrow C_{0}(Y, F)$ is well defined and bounded by Proposition 2.1.

In this section, we first consider the case where the disjointness preserving linear operator $T$ is completely continuous. The main result is in the following.

Theorem 3.1. Let $T$ be a bounded disjointness preserving linear operator from $C_{0}(X, E)$ into $C_{0}(Y, F)$. Then the following are equivalent.

(i) $T$ is completely continuous.

(ii) There are a sequence $\left\{x_{n}\right\}_{n}$ of distinct points in $X$ and a norm null and mutually disjoint sequence $\left\{h_{n}\right\}_{n}$ such that

$$
T f=\sum_{n} \delta_{x_{n}} \otimes h_{n}(f) \quad \text { for all } f \in C_{0}(X, E),
$$

where each $h_{n}: Y \rightarrow B(E, F)$ is continuous and vanishes at infinity in the strong operator topology and is uniformly completely continuous.

To prove this theorem, we need the following results. Let us start with an elementary one.

Lemma 3.2. Let $f_{n}$ be in $C_{0}(X, E)$ with $\left\|f_{n}\right\|=1$. If the $f_{n}$ are mutually disjoint, then $f_{n} \rightarrow 0$ weakly. 
Note that the operator $T$ carries the form in (1). We shall characterize the properties of $h$ and $\varphi$ in the following lemmas.

Lemma 3.3. Let $x_{n}$ be distinct points in $\varphi\left(Y^{\prime}\right)$ and $y_{n}$ in $Y^{\prime}$ such that $\varphi\left(y_{n}\right)=x_{n}$. Then $\lim _{n \rightarrow \infty}\left\|h\left(y_{n}\right)\right\|=0$.

Proof. We may assume on the contrary that there were an $\epsilon>0$ and a sequence $\left\{e_{n}\right\}_{n}$ in $E$ with $\left\|e_{n}\right\|=1$ such that $\left\|h\left(y_{n}\right)\left(e_{n}\right)\right\| \geq \epsilon$ for all $n \in \mathbb{N}$. We discuss the following two cases.

CAsE I. Suppose that every neighborhood $V$ of $x_{1}$ contains all but finitely many of the $x_{n}$. That is, $x_{1}$ is the limit of $\left\{x_{n}\right\}_{n}$. If $z$ is a cluster point of $\left\{x_{n}\right\}_{n}$ in $X \cup\{\infty\}$, then each neighborhood of $z$ contains infinitely many $x_{n}$ and thus intersects with every neighborhood of $x_{1}$. Since $X$ is Hausdorff, we have $x_{1}=z$.

Now, let $V_{n}$ be a compact neighborhood of $x_{n}$ such that $V_{n} \cap V_{m}=\emptyset$ for all $n, m \geq 2$ and $n \neq m$. Choose $f_{n} \in C_{0}(X, E)$ such that $\operatorname{coz}\left(f_{n}\right) \subseteq V_{n}, f_{n}\left(x_{n}\right)=e_{n}$ and $\left\|f_{n}\right\|=1$. Then $\left\{f_{n}\right\}_{n=2}^{\infty}$ is mutually disjoint and, by Lemma $3.2, f_{n} \rightarrow 0$ weakly. Since $T$ is completely continuous, we have $T f_{n} \rightarrow 0$ in norm. But

$$
\left\|T f_{n}\right\| \geq\left\|T f_{n}\left(y_{n}\right)\right\|=\left\|h\left(y_{n}\right)\left(f_{n}\left(\varphi\left(y_{n}\right)\right)\right)\right\|=\left\|h\left(y_{n}\right)\left(e_{n}\right)\right\| \geq \epsilon,
$$

a contradiction.

CASE II. Suppose there exists a compact neighborhood $V_{1}$ of $x_{1}$ such that there are infinitely many $x_{n}$ outside $V_{1}$. Passing to a subsequence if necessary, we can assume that $V_{1}$ contains $x_{1}$ but not $x_{2}, x_{3}, \ldots$ Analogously, in view of CASE I, we may assume that for each $x_{n}$ there exists a compact neighborhood $V_{n}$ of $x_{n}$ containing no other $x_{m}$. Indeed, we can assume that $V_{n} \cap V_{m}=\emptyset$ whenever $n \neq m$. Proceeding as in CASE I, we will get a contradiction again.

Lemma 3.4. For each $x$ in $\varphi\left(Y^{\prime}\right)$, we have that $\varphi^{-1}(x)$ is an open subset of $Y$.

Proof. Suppose that $\varphi^{-1}(x)$ was not open in $Y$. Then $\varphi^{-1}(x)$ was not relatively open in the open set $Y^{\prime}$. In particular, $\varphi^{-1}(x)$ contains a point $y$ not interior to $\varphi^{-1}(x)$. That is, there exists a net $\left\{y_{\lambda}\right\}_{\lambda}$ of $Y^{\prime}$ such that $y_{\lambda} \in Y^{\prime} \backslash \varphi^{-1}(x)$ and $y_{\lambda} \rightarrow y$ in $Y^{\prime}$. Then $\lim _{\lambda \rightarrow \infty} h\left(y_{\lambda}\right)=h(y) \neq 0$ in the strong operator topology. We may assume that there is an $\epsilon>0$ and $e \in E$ with $\|e\|=1$ such that $\left\|h\left(y_{\lambda}\right)(e)\right\|>\epsilon$ for all $\lambda$. By Lemma 3.3, the range of the net $\left\{x_{\lambda}\right\}_{\lambda}=\left\{\varphi\left(y_{\lambda}\right)\right\}_{\lambda}$ consists of only finitely many points in $X$. However, $x_{\lambda}=\varphi\left(y_{\lambda}\right) \rightarrow \varphi(y)=x$. Hence, $x_{\lambda}=x$ for all $\lambda$ eventually, a contradiction. Therefore, $\varphi^{-1}(x)$ is open in $Y$.

For each $x$ in $\varphi\left(Y^{\prime}\right)$, let $Y_{x}=\left\{y \in Y^{\prime}: \varphi(y)=x\right\}=\varphi^{-1}(x)$. Then $Y^{\prime}=$ $\bigcup_{x \in \varphi\left(Y^{\prime}\right)} Y_{x}$ is a disjoint union.

Corollary 3.5. Each $Y_{x}$ is closed and open in $Y^{\prime}$.

Let $h_{x}=\chi_{Y_{x}} \cdot h$, where $\chi_{Y_{x}}$ is the characteristic function of $Y_{x}$. Note that $h_{x}$ and $h_{x^{\prime}}$ are disjoint whenever $x \neq x^{\prime}$ in $\varphi\left(Y^{\prime}\right)$.

Corollary 3.6. For each $x \in \varphi\left(Y^{\prime}\right)$, the operator $h_{x}$ can be continuously extended to $Y \cup\{\infty\}$ in the strong operator topology by setting $\left.h_{x}\right|_{Y_{\infty}}=0$.

Proof. By Corollary 3.5, we have that $h_{x}$ is continuous on $Y^{\prime}$ in the strong operator topology. Let $\left\{y_{\lambda}\right\}_{\lambda}$ be a net in $Y_{x}$ such that $y_{\lambda} \rightarrow y_{0}$ for some $y_{0} \in Y_{\infty}$. If $h_{x}\left(y_{\lambda}\right)$ did not converge to 0 in the strong operator topology, then there is an $\epsilon>0$ and 
$e$ in $E$ such that $\left\|h_{x}\left(y_{\lambda}\right)(e)\right\|=\left\|h\left(y_{\lambda}\right)(e)\right\|>\epsilon$ for all $\lambda$. For each $f$ in $C_{0}(X, E)$ with $f(x)=e$ in $E$, we have

$$
\left\|T f\left(y_{\lambda}\right)\right\|=\left\|h\left(y_{\lambda}\right)\left(f\left(\varphi\left(y_{\lambda}\right)\right)\right)\right\|=\left\|h\left(y_{\lambda}\right)(f(x))\right\|>\epsilon \quad \text { for all } \lambda .
$$

Hence $\left\|T f\left(y_{0}\right)\right\| \geq \epsilon$. But $y_{0} \in Y_{\infty}$, and so we have that $T f\left(y_{0}\right)=0$. It is a contradiction. Therefore, $h_{x}$ can be extended continuously to $Y \cup\{\infty\}$ in the strong operator topology by setting $\left.h_{x}\right|_{Y_{\infty}}=0$.

Lemma 3.7. For each $n=1,2, \ldots$, the set $\left\{x \in \varphi\left(Y^{\prime}\right): \sup _{y \in Y_{x}}\left\|h_{x}(y)\right\|>1 / n\right\}$ is finite. Thus, $\varphi\left(Y^{\prime}\right)$ is a countable set.

Proof. Suppose our assertion were not true. Then there are distinct $x_{1}, x_{2}, \ldots$ in $\varphi\left(Y^{\prime}\right)$ such that $\sup _{y \in Y_{x_{k}}}\left\|h_{x_{k}}(y)\right\|>1 / n$ for all $k$. Let $y_{k} \in Y^{\prime}$ such that $\left\|h_{x_{k}}\left(y_{k}\right)\right\|>1 / n$ and thus $\varphi\left(y_{k}\right)=x_{k}$ for each $k$. But by Lemma 3.3, we have $\lim _{k \rightarrow \infty}\left\|h_{x_{k}}\left(y_{k}\right)\right\|=0$, a contradiction. Hence, the set $\left\{x \in \varphi\left(Y^{\prime}\right)\right.$ : $\left.\sup _{y \in Y_{x}}\left\|h_{x}(y)\right\|>1 / n\right\}$ is finite. Consequently,

$$
\varphi\left(Y^{\prime}\right)=\bigcup_{n=1}^{\infty}\left\{x \in \varphi\left(Y^{\prime}\right): \sup _{y \in Y_{x}}\left\|h_{x}(y)\right\|>1 / n\right\}
$$

is countable.

Now we are ready to prove Theorem 3.1.

Proof of Theorem 3.1. Suppose $T$ is completely continuous. In view of Lemma 3.7, we can write $\varphi\left(Y^{\prime}\right)=\left\{x_{1}, x_{2}, \ldots\right\}$. Each $Y_{n}=\varphi^{-1}\left(x_{n}\right)$ is relatively open and closed in the open set $Y^{\prime}$ by Lemma 3.4 and Corollary 3.5. For each $n \in \mathbb{N}$, let $h_{n}=\chi_{Y_{n}} \cdot h$. Then $h_{n}$ is continuous on $Y$, vanishes at infinity in the strong operator topology by Corollary 3.6, and $\left\|h_{n}\right\| \rightarrow 0$ by Lemma 3.7. Note that the $h_{n}$ are mutually disjoint.

Observe that for all $x_{n}$ in $\varphi\left(Y^{\prime}\right)$, we have $h(y)(f(\varphi(y)))=h_{n}(y)\left(f\left(x_{n}\right)\right)$, since $\varphi$ is constantly $x_{n}$ on $Y_{n}$. Hence, for each $y \in Y^{\prime}$ and $f \in C_{0}(X, E)$,

$$
T f(y)=h(y)(f(\varphi(y)))=\sum_{y \in Y_{n}} h(y)(f(\varphi(y)))=\sum_{n} h_{n}(y)\left(f\left(x_{n}\right)\right) .
$$

By Corollary 3.6, we can write

$$
T f=\sum_{n=1}^{\infty} \delta_{x_{n}} \otimes h_{n}(f) \quad \text { for all } f \in C_{0}(X, E) .
$$

In fact, since $\left\{h_{n}\right\}_{n}$ is mutually disjoint and converges to 0 in norm, the sum $T=\sum_{n} \delta_{x_{n}} \otimes h_{n}$ converges in the operator norm. Moreover, it is clear that $\delta_{x_{n}} \otimes h_{n}$ is completely continuous, and we have that each $h_{n}$ is uniformly completely continuous on $Y$ by Lemma 2.3.

Conversely, since each $h_{n}$ is uniformly completely continuous on $Y$, we have that $\delta_{x_{n}} \otimes h_{n}$ is completely continuous by Lemma 2.3. As we know that the norm limit of completely continuous operators is completely continuous (e.g. [10, p. 301]), hence $T=\sum_{n} \delta_{x_{n}} \otimes h_{n}$ is completely continuous.

For compact disjointness preserving linear operators, there are parallel results such as the following. 
Lemma 3.8. Suppose the disjointness preserving operator $T: C_{0}(X, E) \rightarrow$ $C_{0}(Y, F)$ is compact with the form in (1). Then $h_{x}$ can be extended to $Y \cup\{\infty\}$ as a norm continuous operator by setting $\left.h_{x}\right|_{Y_{\infty}}=0$ for each $x$ in $\varphi\left(Y^{\prime}\right)$.

Proof. By the compactness of $T$, we have that $h$ is continuous and vanishes at infinity in the uniform operator topology [3]. By Corollary 3.5, $h_{x}$ is continuous on $Y^{\prime}$ in the norm topology. Let $\left\{y_{\lambda}\right\}_{\lambda}$ be a net in $Y_{x}$ such that $y_{\lambda} \rightarrow y_{0}$ for some $y_{0}$ in $Y_{\infty}$. If $h_{x}\left(y_{\lambda}\right)$ did not converge to 0 in norm, then, by passing to a subnet, we could assume that $\left\|h\left(y_{\lambda}\right)\right\|=\left\|h_{x}\left(y_{\lambda}\right)\right\|>\epsilon$ for some $\epsilon>0$. Then there would be a net $\left\{e_{\lambda}\right\}_{\lambda}$ in $E$ such that $\left\|e_{\lambda}\right\|=1$ and $\left\|h\left(y_{\lambda}\right)\left(e_{\lambda}\right)\right\|>\epsilon$. Let $\left\{f_{\lambda}\right\}_{\lambda}$ be in $C_{0}(X, E)$ such that $f_{\lambda}(x)=e_{\lambda}$ and $\left\|f_{\lambda}\right\|=1$. By the compactness of $T$ and passing to a subnet, we have $T f_{\lambda} \rightarrow g$ in norm for some $g \in C_{0}(Y, F)$. More precisely, there is a $\lambda_{0}>0$ such that

$$
\left\|h(y)\left(e_{\lambda}\right)-g(y)\right\|=\left\|T f_{\lambda}(y)-g(y)\right\|<\epsilon / 2 \quad \text { for all } y \in Y \text { and } \lambda \geq \lambda_{0} .
$$

This implies that $\left\|g\left(y_{\lambda}\right)\right\|>\epsilon / 2$ for all $\lambda \geq \lambda_{0}$, and then $\left\|g\left(y_{0}\right)\right\| \geq \epsilon / 2$. On the other hand, it follows from $y_{0} \in Y_{\infty}$ that $T f_{\lambda}\left(y_{0}\right)=0$ for all $\lambda$. We have $g\left(y_{0}\right)=0$, a contradiction. Hence, $h_{x}$ can be continuously extended to $Y \cup\{\infty\}$ in the uniform operator topology by setting $\left.h_{x}\right|_{Y_{\infty}}=0$.

Theorem 3.9. Let $T: C_{0}(X, E) \rightarrow C_{0}(Y, F)$ be a bounded disjointness preserving linear operator. Then the following are equivalent.

(i) $T$ is compact.

(ii) There are a sequence $\left\{x_{n}\right\}_{n}$ of distinct points in $X$ and a norm null and mutually disjoint sequence $\left\{h_{n}\right\}_{n}$ such that

$$
T=\sum_{n} \delta_{x_{n}} \otimes h_{n}
$$

where each $h_{n}: Y \rightarrow B(E, F)$ is continuous and vanishes at infinity in the uniform operator topology, and $h_{n}(y)$ is compact for every $y \in Y$.

Proof. By using Lemmas 2.2 and 3.8, the theorem follows from similar arguments as in the proof of Theorem 3.1.

\section{REFERENCES}

[1] Yu. A. Abramovich, Multiplicative representation of disjointness preserving operators, Indag. Math. 45, no. 3 (1983), 265-279. MR718068 (85f:47040)

[2] Yu. A. Abramovich, A. I. Veksler and A. V. Koldunov, On operators preserving disjointness, Soviet Math. Dokl. 248 (1979), 1033-1036. MR553919 (81e:47034)

[3] Jor-Ting Chan, Operators with the disjoint support property, J. Operator Theory 24 (1990), 383-391. MR1150627 (93c:47035)

[4] W. A. Feldman and J. F. Porter, Operators on Banach lattices as weighted compositions, J. London Math. Soc. 33 (1986), 149-156. MR829395 (87j:47054)

[5] J. J. Font and S. Herández, On separating maps between locally compact spaces, Arch. Math. (Basel) 63 (1994), 158-165. MR1289298 (95k:46083)

[6] J. J. Font, Disjointness preserving mappings between Fourier algebras, Colloq. Math. 77 (1998), 179-187. MR1629037 (99g:43002)

[7] J. E. Jamison and M. Rajagopalan, Weighted composition operator on $C(X, E)$, J. Operator Theory 20 (1988), 307-317. MR960982 (90b:47052)

[8] K. Jarosz, Automatic continuity of separating linear isomorphisms, Canad. Math. Bull. 33 (1990), 139-144. MR1060366 (92j:46049)

[9] Ying-Fen Lin and Ngai-Ching Wong, The structure of compact disjointness preserving operators of continuous functions, submitted. 
[10] Y.-C. Wong, Introductory theory of topological vector spaces, Marcel Dekker, New York, 1992. MR1198892 (94c:46003)

Department of Management Sciences, no. 1, Wei-Wu Rd., Military Academy, FengSHAN KAOHSIUNG 830, TAIWAN

E-mail address: jeangjs@mail.cma.edu.tw

Department of Mathematics, National Hualien University of Education, Hua-Lien, 970, TAIWAN

E-mail address: linyf@mail.nhlue.edu.tw 